\title{
Contribuciones de la Escuela Agrícola Panamericana a la Salud Animal
}

\author{
John J. Hincapié ${ }^{1}$
}

Resumen. La salud animal es uno de los pilares en la producción agropecuaria, y por tanto cobra especial importancia en los procesos de enseñanza, investigación y transferencia de tecnología. La Escuela Agrícola Panamericana (EAP) se ha caracterizado desde sus comienzos, en la década del 40, por velar por el buen desempeño en sanidad animal en sus unidades de producción y en la enseñanza de las buenas prácticas sanitarias a sus estudiantes de más de 16 países latinoamericanos. Durante este tiempo, han brindado sus servicios a la institución veterinarios de origen español, hondureños, peruano y colombiano, quienes siempre han estado comprometidos con los valores y la misión zamorana del Aprender-Haciendo bajo el lema El trabajo todo lo vence. Gracias a todos ellos y al esfuerzo en conjunto con la institución, el personal de campo, los estudiantes, empresas privadas y otras instituciones nacionales e internacionales, la EAP se a posicionado como líder latinoamericano en la investigación agropecuaria. Son muchos los trabajos de investigación que se han desarrollado durante estos años en la áreas de bovinos (nutrición, alimentación, sanidad, reproducción controlada como la inseminación artificial, transferencia de embriones, sincronización del celo y la ovulación; anestesioterapia y ozonoterapia), porcinos (alimentación y nutrición, reproducción y sanidad), aves (nutrición, alimentación, instalaciones, manejo y sanidad), peces (nutrición, alimentación, manejo, reproducción y sanidad) y equinos (reproducción y sanidad), brindando con ellos soluciones a las problemáticas de campo que afrontan los productores, no solo en Honduras, sino en otros países como México, El Salvador, Nicaragua, Colombia y Venezuela, contribuyendo con ello al progreso socioeconómico.

Palabras clave: Dedicación, disciplina, extensión agropecuaria, enseñanza, ganadería, veterinaria.

\section{Contributions of the Escuela Agrícola Panamericana to Animal Health}

\begin{abstract}
Animal health is a foundation stone of agricultural production, and thus is particularly important in the development of teaching, research and technology transfer. The Escuela Agrícola Panamericana (EAP) has been known since its beginnings in the 40's for its good performance in animal production units and in teaching of good animal health practices to students from over 16 Latin American countries. During this time, veterinarians from Spain, Honduras, Peru, and Colombia have offered their services to the institution. They all have always been committed to the values and mission of Zamorano's Learning by Doing motto "work conquers all". Thanks to all of them and the joint effort with the institution, field staff, students, private companies, and national and international institutions, the EAP is a Latin-American leader in agricultural research. There are many research documents that have been developed over the years in the areas of cattle (nutrition and feeding, health, controlled reproduction and artificial insemination, embryo transfer, synchronization of estrus and ovulation, anesthesia-therapy, and ozone-therapy), swine (nutrition and feeding, reproduction, and health), poultry (nutrition, feeding, and health), fish (nutrition, feeding, reproduction, and health), and equine (reproduction and health) which have provided solutions to different problems faced by producers not only in Honduras but also countries such as Mexico, El Salvador, Nicaragua, Colombia, and Venezuela; therefore contributing to their economic progress.
\end{abstract}

Keywords: Dedication, discipline, agricultural extension, education, livestock, veterinary medicine.

\section{Introducción}

La Escuela Agrícola Panamericana (EAP), también conocida como Zamorano, desde sus comienzos a velado por salud animal, y por lo tanto no ha sido ajena a la importancia que representa y su efecto directo en los indicadores económicos y en la salud humana. Es así como en 1955, el Dr. Ramón Peruga, de origen español, comenzó con la labor veterinaria en la institución, velando por sus unidades de producción, enseñando en ese entonces los principios básicos de la sanidad animal (dictó la clase de Elementos de la Medicina Veterinaria) y el cuidado veterinario, así como haciendo proyección con los

\footnotetext{
${ }^{1}$ Escuela Agrícola Panamericana, Zamorano, Honduras, email jhincapie@ zamorano.edu
} 
vecinos. El programa de inseminación artificial en la Escuela Agrícola Panamericana empezó en 1959 y así tuvo el primer departamento de ganadería en América Latina que enseñó el uso del semen congelado importado.

En los años 60's, laboró el Dr. Jorge Reyes, salvadoreño y quien se desempeñó como profesor asistente en el Departamento de Ganadería y Médico Veterinario. En 1961 ingresó el Dr. Francisco Matamoros, hondureño y graduado de Zamorano en 1948 y quien posteriormente estudió Medicina Veterinaria; él laboró por cerca de tres años, tiempo en el cual desarrolló una magnífica labor de educación y extensión agropecuaria (Figuras 1 y 2). Posteriormente ingresó el Dr. Carlos R. Pinel (EAP 1965), hondureño, quien estuvo al frente de la sección de veterinaria por espacio de dos años, retirándose de la institución en 1964. Este mismo año ingresó el Dr. Guillermo Torres Yufra, peruano, y quien laboró en la institución cerca de 31 años (1964-1995) (Figuras 3 y 4). Durante estos años iniciales, la labor veterinaria fue enfocada en mantener el bienestar de los animales de las unidades de producción, dictar el aprender haciendo a los estudiantes y servir de apoyo a estudiantes egresados zamoranos que venían a realizar sus trabajos de posgrado (maestrías y doctorados).

A partir de 1996, con el ingreso del Dr. John Jairo Hincapié, colombiano, se inició un proceso de investigación y desarrollo de trabajos de graduación o tesis en el área de sanidad y reproducción animal, en bovinos, porcinos, peces, equinos y aves (Figuras 5, 6 y 7). La gran mayoría de estas investigaciones han estado enfocadas a buscar soluciones prácticas a las problemáticas de los productores, logrando mejorar sus sistemas de producción animal.

Es de suma importancia recalcar el papel protagónico que tiene el desarrollo de la tesis como requisito de graduación para optar al título de Ingeniero Agrónomo; este protagonismo radica en el desarrollo de investigaciones de alta calidad, enfocadas a dar soluciones prácticas y aplicables al campo agropecuario, no solamente local, sino latinoamericano, lo que ubica a la Escuela Agrícola Panamericana como líder en la educación agropecuaria y la investigación aplicada.

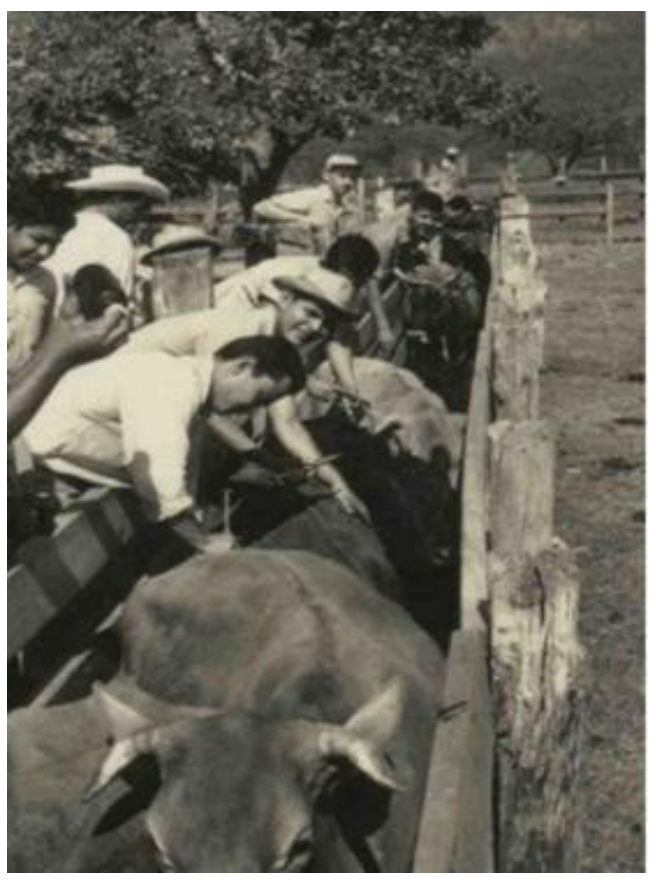

Figura 1. El Dr. Francisco Matamoros en la práctica de ganadería con los estudiantes de la Escuela Agrícola Panamericana.

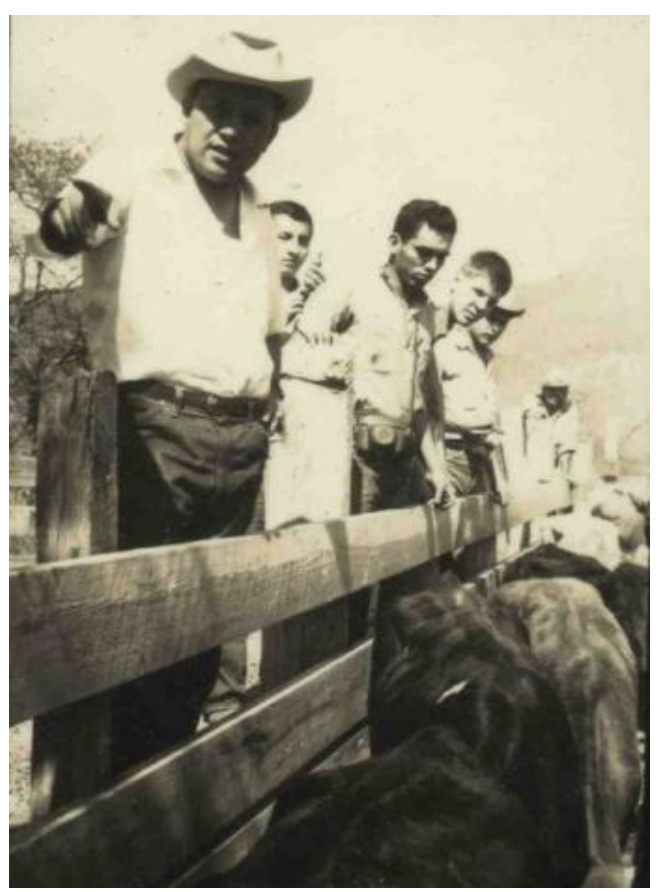

Figura 2. Un grupo de estudiantes con el Dr. Francisco Matamoros en la unidad de ganadería. 


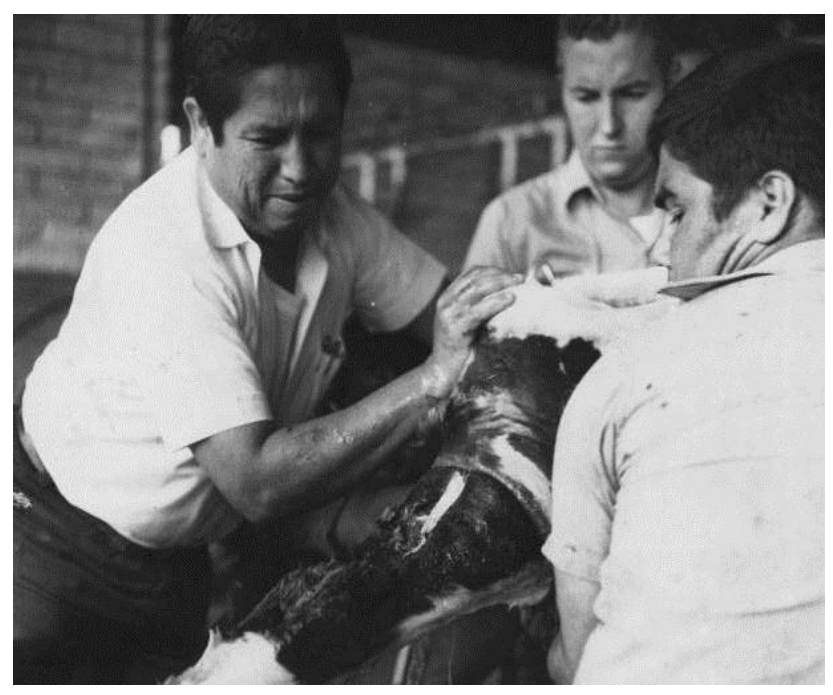

Figura 3. El Dr. Guillermo Torres Yufra atiende un parto distócico con estudiantes de la Escuela Agrícola Panamericana en la unidad de ganado lechero.

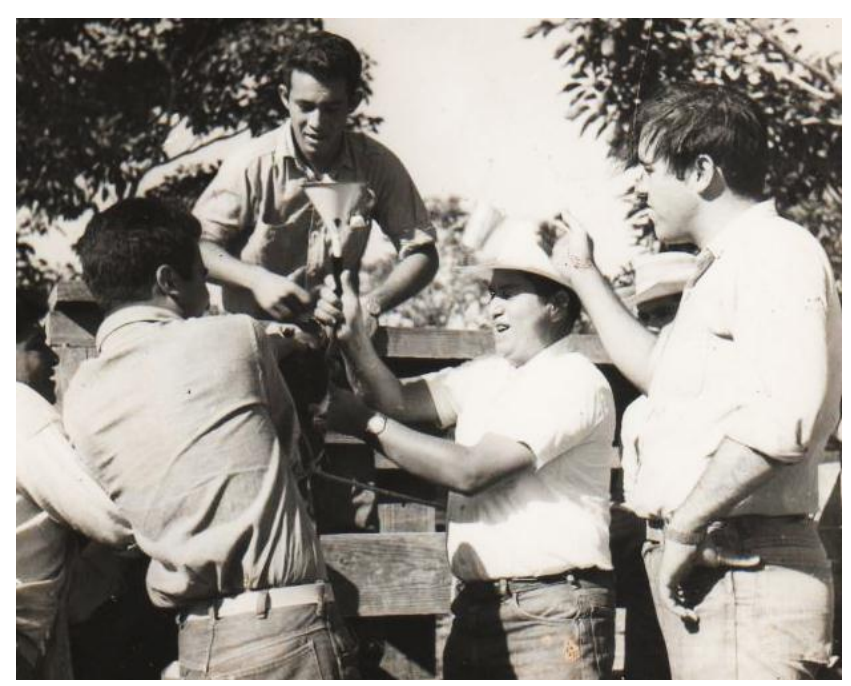

Figura 4. El Dr. Guillermo Torres Yufra administrando medicamentos en una práctica con estudiantes de la Escuela Agrícola Panamericana.

\section{Investigaciones}

Ganado Lechero y de Carne. Al realizar una remembranza de los aportes científicos de Zamorano a la sanidad animal, se pueden citar trabajos desde 1994 en los cuales se desarrollaron investigaciones que brindaron aportes sobre la metritis y su efecto sobre el comportamiento reproductivo (Mercadal Zapata 1994; Medina Ortega 1999), así como trabajos sobre el control biológico de la mosca en las instalaciones agropecuarias (Gonzáles Monroy 1994); otras investigaciones sobre micotoxinas (Morán Berlioz 1998) y modelos de capacitación en sanidad animal (Gallozzi Cálix 1998) han brindado valiosa información al sector agropecuario.

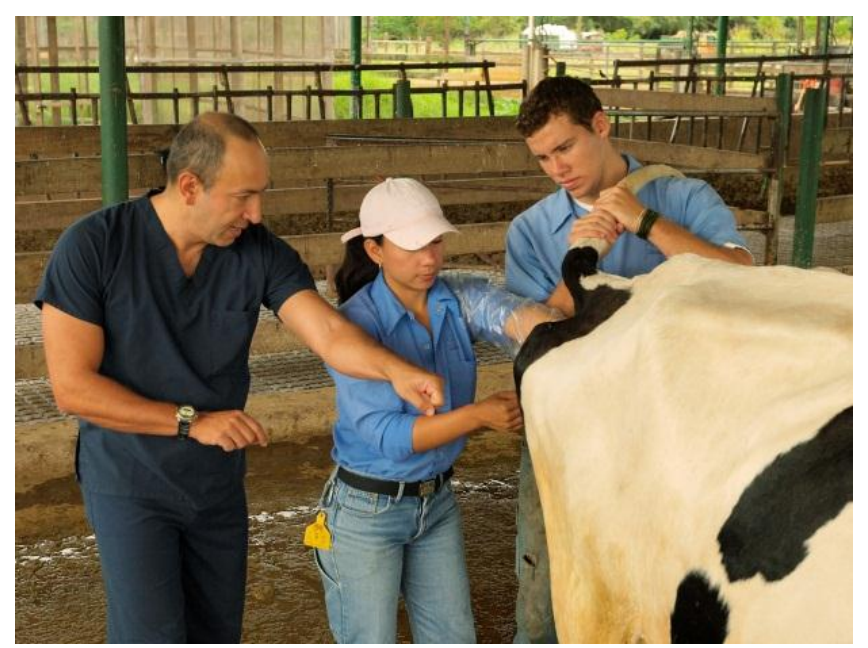

Figura 5. El Dr. John Jairo Hincapié S. enseñando la técnica de inseminación artificial a estudiantes de la Escuela Agrícola Panamericana.

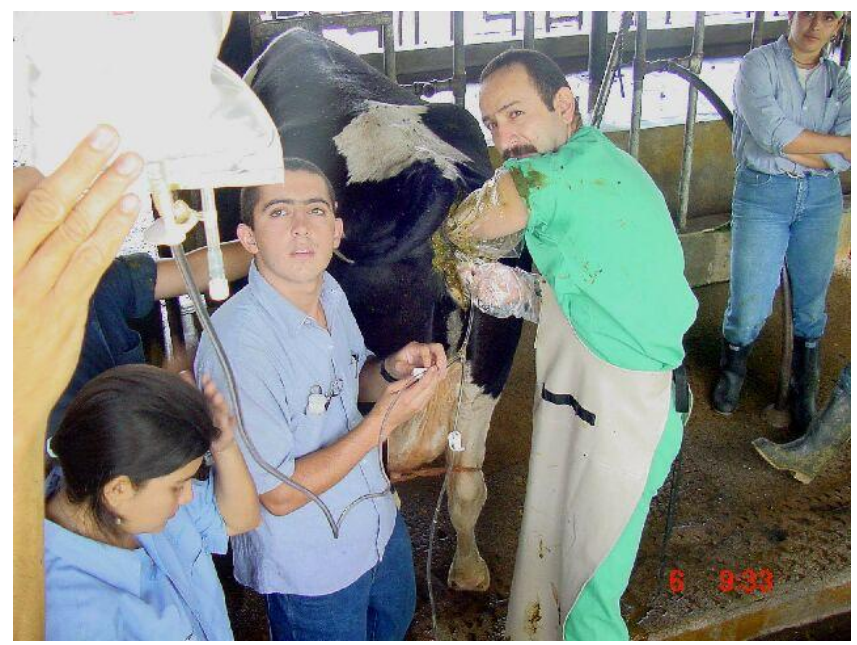

Figura 6. El Dr. John Jairo Hincapié S. enseñando la técnica de transferencia de embriones en bovinos. 


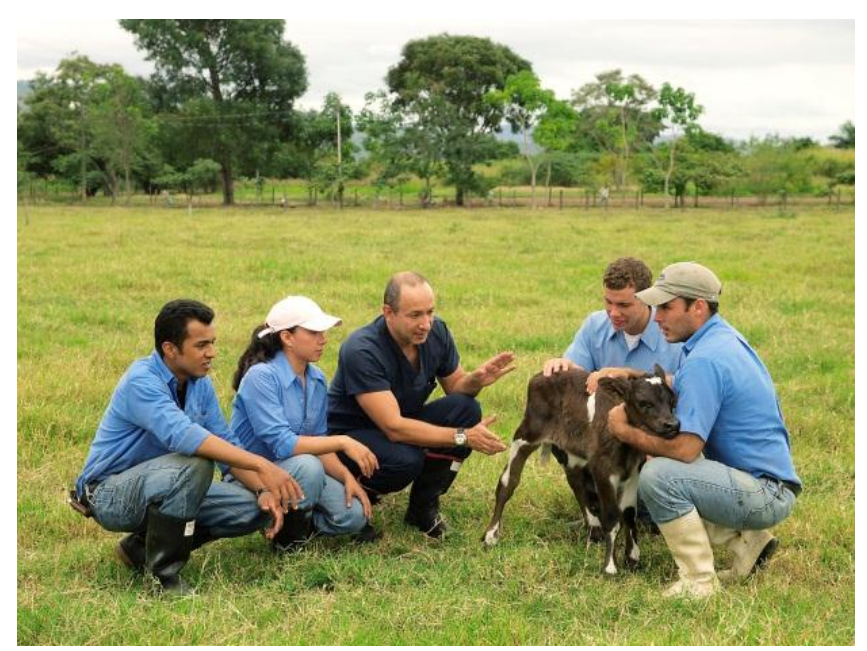

Figura 7. El Dr. John Jairo Hincapié impartiendo una charla a los estudiantes de la Escuela Agrícola Panamericana sobre el levante de terneras

Otras investigaciones han puesto en evidencia la importancia de una buena alimentación en el ganado lechero y sus efectos sobre el desarrollo, la salud ruminal, metabólica y reproductiva (López Rodríguez 2000; Borjas Guifarro 2004; Montalván Ruíz 2004; Sierra Méndez 2005; Revelo Saltos 2005; Fortín Cabrera y Perdomo Carbajal 2009; Matamoros Hernández y Moreno Rajo 2009. Además, investigaciones en epidemiología y parasitología (Rodríguez 2000; Andrade Foronda 2002; Ponce Velásquez y Marín Elías 2002; Sobalvarro Acosta 2003; Sosa Cuevas 2009; Gutiérrez Luna y Borjas Carvajal 2010) han dejado claro la importancia de mantener estrictos planes de sanidad animal, los cuales incluyen programas integrados de manejo y control de garrapatas, desparasitaciones y vacunaciones contra enfermedades bacterianas o virales.

Otra línea de investigación en Zamorano es el uso de las terapias alternativas como la anestesioterapia y la ozonoterapia en los tratamientos de las patologías reproductivas (Ortega Obando 2003; Morales del Cid 2004; Osorio Estévez 2004; Portillo Sandoval y Villacís Carrera 2005; Perla Barrera 2005; Freire Coba 2011) demostrando que con la aplicación de estas terapias se reducen en más de un $60 \%$ los costos de los tratamientos de afecciones como la retención de placenta, piómetras, metritis, endometritis, además de una notable disminución en el tiempo de recuperación del paciente y con ello una reincorporación más rápida a la reproducción y la producción.

El uso de la tecnología ultrasónica ha permitido desarrollar investigaciones sobre las técnicas de diagnóstico precoz en la gestación en bovinos (Herrarte Abascal 2005), dinámica folicular (Vergara Icaza 2005) y manipulación uterina (Pazmiño Haro 2005), permitiendo mejorar los indicadores reproductivos en los hatos, al detectar en forma temprana las vacas vacías y poderlas reenrolar rápidamente en los lotes de inseminación. Así, se disminuyen los días improductivos de las vacas, se determinan patologías como quistes ováricos, endometritis y anomalías de la gestación (momias, reabsorciones embrionarias, tumores), logrando corregirlas en forma temprana y mejorar los indicadores productivos.

A medida que se avanzaba en las líneas de investigación, se fueron desarrollando trabajos en conjunto con otras instituciones de Venezuela, Colombia, Nicaragua, México, El Salvador y España, con el objetivo de buscar soluciones a las problemáticas que afrontan los productores agropecuarios en dichos países, colocando a Zamorano como líder en la investigación agropecuaria a nivel tropical (Carvajal Núñez y Pérez Perdomo 2007; Orellana Banegas y Peralta Peralta 2007).

La manipulación endocrina del ciclo estral bovino ha sido uno de los pilares en la reproducción animal para mejorar los indicadores reproductivos y los estándares de producción en carne y leche. El uso de dispositivos intravaginales de liberación lenta de progesterona para sincronizar celos y la ovulación en vacas cíclicas, acíclicas, vaquillas de leche y de carne, en la estimulación del reinicio de la actividad ovárica posparto, reutilizados, combinados con diferentes esquemas y dosis hormonales, han sido ampliamente estudiados en Zamorano (Benalcázar Sandoval y Valencia Villareal 2005; Vélez Pérez 2005; Barillas Flores y Carballo Carias 2007; Canales Matamoros 2007; Flaquer Borbón 2007; Pérez de la Ossa 2007; Villavicencio Celí 2007; Bueno López y Dunn Barragán 2008; Guevara Florentino 2008; Vázcones Miño y Ortega González 2009; Espinal Tercero y García Mejía 2009; Diéguez Juárez y Escobar Cerrato 2009; Espinal Méndez y Cedeño Orocú 2009; 
Menjívar Polanco y Barahona Rosales 2009; Pinto Portillo y Chacón García 2009; Martínez Pittí y Sierra Sagastume 2010; Ayala Constante y Castillo Rosa 2010; Cerrud Amores y Delgado 2010; Velázquez Mejía y Vélez Bravo 2011; Rodríguez Calvo y Lobo Rosa 2011; Acosta Maldonado y Rodríguez Sánchez 2011).

Todas estas investigaciones endocrina del ciclo estral bovino han ayudado a los ganaderos a incorporar programas de inseminación artificial, implementar programas de mejoramiento genético, introducir nuevas razas que les permiten obtener cruces con las mejores características de dos o más razas, reincorporar lotes de vacas acíclicas a la reproducción, controlar las épocas de montas y de partos, uniformizar los destetes para concentrar las labores en determinadas épocas del año, optimizando así el uso del tiempo en la finca y distribuir los partos en ganado de leche a través del año acorde con la demanda de leche por las plantas procesadoras.

La biotecnología (transferencia de embriones, la criopreservación de semen bovino y el estudio de varios diluyentes) ha aportado valiosa información del comportamiento de los gametos masculinos y femeninos bajo diferentes condiciones de manejo y criopreservación. Esto ha permitido a los productores tener procedimientos para conservar semen de sus mejores sementales y garañones, acortar el intervalo intergeneracional con la transferencia de embriones y aprovechar al máximo el potencial genético de vacas élite. Los productores han logrado obtener hasta más de 20 crías por vaca por año y conservar embriones en criopreservación de vacas élite para su uso futuro (Morán Ramírez y Díaz Lezama 2006; Eveline Padilla y Vaquero Castañeda 2006; Alban García y Olmedo Charro 2006; Carvajal Córdova 2006; Raudales Zúñiga y Cerrato Zelaya 2006; Betancourth y Cáceres Gutiérrez 2011); además, se ha investigado la inducción de la lactancia en vaquillas y vaquillonas con problemas reproductivos, lo cual permite al productor aprovechar estos animales antes de ser descartados del hato (David Lagos 2007).

Cabras y Ovejas. En las otras especies, como cabras y ovejas, también se han desarrollado investigaciones sobre la salud animal, las cuales han aportado valiosos conocimientos a los productores principalmente en el control de endoparásitos utilizando productos orgánicos, amigables con el ambiente, de fácil adquisición y de muy bajo costo e inclusive en ocasiones puede estar disponible en los potreros de las fincas (Rodas Lagos 1997).

Cerdos. La actividad porcina es una de las más importantes en la economía internacional, por esto Zamorano ha orientado muchos de sus recursos a la investigación en este aspecto. Se han desarrollado investigaciones en sanidad porcina (Mendoza García 2001; Guzmán Calderón 2002; Posas Flores 2002) enfocadas a buscar soluciones a los principales problemas de infecciones bacterianas, virales y parasitarias, con resultados muy positivos y aplicables a la realidad pecuaria.

Otros trabajos en reproducción porcina han caracterizado las líneas de investigación en Zamorano, enfocadas al mejoramiento del comportamiento reproductivo de esta especie con el objetivo primordial de obtener la mayor eficiencia en la producción y con ello incrementar la rentabilidad de la explotación (Fernández Hernández 2005; García Guerrero 2005).

Avicultura. La avicultura ha sido durante décadas una de las industrias más productivas en Latinoamérica y Zamorano como tal, ha sido partícipe activo en los programas de investigación, dentro de los cuales el aspecto sanitario adquiere suma importancia; muchas de estas investigaciones han abordado temas de interés para los avicultores como el control de la coccidiosis, control de parásitos internos y externos, manejo químico y microbiológico de la cama en pollos de engorde (Acosta Jovel e Hidalgo Cuesta 2002; Cruz Huilcamaigua 2002; Espinoza Mora 2003; Ruíz Faz e Ibarra Ruíz 2005).

Peces y Camarones. En sanidad de peces y camarones se han desarrollado investigaciones en el área sanitaria enfocadas a buscar soluciones a la infección por bacterias filamentosas en los cultivos de camarón (Araujo Delgado 1999).

Equinos. La sanidad animal ha tenido un lugar privilegiado en equinos ya que es una especie que, aparte de despertar un gran sentimiento en sus propietarios como animales de compañía, son 
animales involucrados en la producción pecuaria como animales de tiro y vaquería. Por su importancia en proceso productivo, se han desarrollado investigaciones en parasitología (Guerrero Soria 2006) $\mathrm{y}$ en acondicionamiento con terapias alternativas (Guevara López 2007), logrando resultados valiosos y de aplicables por los dueños de los equinos.

\section{Conclusiones}

La salud animal ha sido, es y seguirá siendo uno de los principales pedestales en la enseñanza e investigación en Zamorano, contribuyendo con ello a buscar soluciones a las dificultades que afronta el productor en el sector agropecuario con el objetivo de aumentar los rendimientos y con ello la calidad de vida de todos los involucrados en las cadenas productivas agropecuarias.

Actualmente, el laboratorio de reproducción animal está dotado con equipos de alta tecnología que permiten desarrollar procesos, investigaciones y las prácticas del aprender haciendo en áreas como la criopreservación de semen bovino, equino y porcino, transferencia de embriones bovinos y su criopreservación y fertilización in vitro en bovinos, garantizando con ello una enseñanza de alta calidad con tecnología de avanzada. Sin embargo, consideramos que lo más importante es la diseminación del conocimiento adquirido en Zamorano a través de nuestros estudiantes de más de 16 países latinoamericanos.

\section{Literatura Citada}

Acosta Maldonado, P.L. y R.J. Rodríguez Sánchez. 2011. Porcentaje de preñez en vacas lecheras sometidas a sincronización del celo y la aplicación de progesterona el día 13 pos-servicio. Proyecto Especial del Programa de Ing. Agr., Zamorano, Honduras, Escuela Agrícola Panamericana. $19 \mathrm{p}$.

Acosta Jovel, C. y M. Hidalgo Cuesta. 2002. Evaluación de Alquernat Zycox ${ }^{\circledR}$ para la prevención de la coccidiosis en pollos de engorde. Proyecto Especial del Programa de Ing. Agr., Zamorano, Honduras, Escuela Agrícola Panamericana. $17 \mathrm{p}$.
Albán García, A. y R. Olmedo Charro. 2006. Efecto de la variación de la temperatura en la curva de enfriamiento $\mathrm{y}$ en el proceso de empaque sobre la calidad biológica del semen bovino. Proyecto Especial del Programa de Ing. Agr., Zamorano, Honduras, Escuela Agrícola Panamericana. $20 \mathrm{p}$.

Andrade Foronda, D. 2002. Estudio epidemiológico de Brucelosis y Leptospirosis en el Litoral Atlántico y el Valle de Aguán. Proyecto Especial del Programa de Ing. Agr., Zamorano, Honduras, Escuela Agrícola Panamericana. $18 \mathrm{p}$.

Araujo Delgado, N. 1999. Uso de Cloramina T para el control de bacterias filamentosas (Leucothrix mисоr) en post-larvas de camarón (Penaeus vannamei). Proyecto Especial del Programa de Ing. Agr., Zamorano, Honduras, Escuela Agrícola Panamericana. $19 \mathrm{p}$.

Ayala Constante, D.C. y O.J. Castillo Rosa. 2010. Efecto de la aplicación de GnRH al momento de la inseminación artificial en vacas lecheras implantadas con dispositivos intravaginales. Proyecto Especial del Programa de Ing. Agr., Zamorano, Honduras, Escuela Agrícola Panamericana . $14 \mathrm{p}$.

Barillas Flores, M. y R. Carballo Carías. 2007. Tasa de preñez en vacas anéstricas tratadas con el dispositivo intravaginal $\mathrm{CIDR}^{\circledR}$ más benzoato de estradiol o cipionato de estradiol y GnRH e inseminadas a celo detectado. Proyecto Especial del Programa de Ing. Agr., Zamorano, Honduras, Escuela Agrícola Panamericana. $18 \mathrm{p}$.

Benalcázar Sandoval, V. y E. Valencia Villareal. 2005. Efecto de un dispositivo intravaginal a base de progestágenos sobre el comportamiento reproductivo en novillas mestizas Brahman en anestro, en el Estado de Zulia, Venezuela. Proyecto Especial del Programa de Ing. Agr., Zamorano, Honduras, Escuela Agrícola. $16 \mathrm{p}$.

Betancourth, J.F. y G. Cáceres Gutiérrez. 2011. Superovulación y transferencia de embriones en vacas lecheras utilizando dos protocolos hormonales. Proyecto Especial del Programa de Ing. Agr., Zamorano, Honduras, Escuela Agrícola. 18 p.

Borjas Guifarro, A. 2004. Relación entre los niveles séricos de colesterol y glucosa ante y posparto con la presencia de trastornos reproductivos durante el puerperio en vacas lecheras en Zamorano. Proyecto Especial del Programa de Ing. Agr., Zamorano, Honduras, Escuela Agrícola Panamericana. 13 p. 
Bueno López, A. y R. Dunn Barragán. 2008. Tasa de preñez en vaquillas anéstricas tratadas con $\mathrm{CIDR}^{\circledR}$ más benzoato de estradiol, cipionato de estradiol o GnRH e inseminadas a celo detectado. Proyecto Especial del Programa de Ing. Agr., Zamorano, Honduras, Escuela Agrícola Panamericana. 16 p.

Canales Matamoros, C. 2007. Efecto de la GnRH $+\mathrm{PGF}_{2} \alpha$ y el dispositivo intravaginal $\mathrm{CIDR}_{\circledast}+\mathrm{ECP}$ en el tratamiento del anestro posparto en vacas lecheras. Proyecto Especial del Programa de Ing. Agr., Zamorano, Honduras, Escuela Agrícola Panamericana. $16 \mathrm{p}$.

Carvajal Núñez, D. y O. Pérez Perdomo. 2007. Evaluación de cuatro frecuencias de alimentación en ganado Holstein, en la comarca Lagunera, Las Lomas, Durango, México. Proyecto Especial del Programa de Ing. Agr., Zamorano, Honduras, Escuela Agrícola Panamericana. 17 p.

Carvajal Córdova, C.G. 2006. Relación entre la prueba de reducción del azul de metileno con la actividad metabólica y los parámetros macro y microscópicos del semen fresco de bovino. Proyecto Especial del Programa de Ing. Agr., Zamorano, Honduras, Escuela Agrícola Panamericana. 18 p.

Cerrud Amores, F. y J.A. Delgado. 2010. Efecto de la sincronización y resincronización de celos sobre el porcentaje de preñez en la raza Brangus. Proyecto Especial del Programa de Ing. Agr., Zamorano, Honduras, Escuela Agrícola Panamericana. 17 p.

Cruz Huilcamaigua, C. 2002. Relación entre el título de anticuerpos maternos y la presencia del virus de Gumboro en la bolsa de Fabricio en pollos de engorde. Proyecto Especial del Programa de Ing. Agr., Zamorano, Honduras, Escuela Agrícola Panamericana. $68 \mathrm{p}$.

David Lagos, K.L. 2007. Inducción de la lactancia con hormonas en vacas y vaquillas con problemas reproductivos. Proyecto Especial del Programa de Ing. Agr., Zamorano, Honduras, Escuela Agrícola Panamericana. $6 \mathrm{p}$.

Diéguez Juárez, A. J. y R.M. Escobar Cerrato. 2009. Efecto de la condición corporal sobre el porcentaje de preñez en vacas sincronizadas con dispositivos intravaginales DIV-B ${ }^{\circledR}$. Proyecto Especial del Programa de Ing. Agr., Zamorano, Honduras, Escuela Agrícola Panamericana. $14 \mathrm{p}$.

EAP (Escuela Agrícola Panamericana). 1965. Informe Anual 1964. s.n.t.
Espinal Tercero, A.G. y B.E. García Mejía. 2009. Efecto de la aplicación de eCG el día ocho del tratamiento con dispositivos intravaginales DIV-B ${ }^{\circledR}$ sobre el porcentaje de preñez en vacas de aptitud lechera con baja condición corporal. Proyecto Especial del Programa de Ing. Agr., Zamorano, Honduras, Escuela Agrícola Panamericana. $15 \mathrm{p}$.

Espinal Méndez, A.M. y M.A. Cedeño Orocú. 2009. Efecto de los dispositivos intravaginales DIV-B ${ }^{\circledR}$ nuevos o usados y retirados el día 8 o 9 sobre los porcentajes de sincronización de celo y preñez en vacas cebuinas. Proyecto Especial del Programa de Ing. Agr., Zamorano, Honduras, Escuela Agrícola Panamericana. $15 \mathrm{p}$.

Espinoza Mora, A. 2003. Efecto de la Ivermectina (Ivercide $^{\circledR}$ ) en el control de parásitos internos y externos en pollo de engorde en Zamorano. Proyecto Especial del Programa de Ing. Agr., Zamorano, Honduras, Escuela Agrícola Panamericana. 15 p.

Eveline Padilla, A. y M. Vaquero Castañeda. 2006. Efecto de la disminución parcial de plasma seminal sobre la calidad biológica del semen bovino poscongelado. Proyecto Especial del Programa de Ing. Agr., Zamorano, Honduras, Escuela Agrícola Panamericana. $18 \mathrm{p}$.

Fernández Hernández, R. 2005. Inmunolocalización del Factor de Crecimiento Similar a la Insulina (IGF-I) y su receptor (IGF-Ir) en el tracto reproductor del verraco. Proyecto Especial del Programa de Ing. Agr., Zamorano, Honduras, Escuela Agrícola Panamericana. $21 \mathrm{p}$.

Flaquer Borbón, J. 2007. Respuesta a la inducción y sincronización del celo con $\mathrm{CIDR}^{\circledR}, \mathrm{GnRH}^{\circledR}$ y $\mathrm{PGF}_{2} \alpha$ en vacas de doble propósito en anestro. Proyecto Especial del Programa de Ing. Agr., Zamorano, Honduras, Escuela Agrícola Panamericana. 15 p.

Fortín Cabrera, A. y J.J. Perdomo Carbajal. 2009. Determinación de la calidad del calostro bovino a partir de la densidad y la concentración de $\operatorname{IgG}$ y de los partos de la vaca y su efecto en el desarrollo de los terneros hasta los 30 días de edad. Proyecto Especial del Programa de Ing. Agr., Zamorano, Honduras, Escuela Agrícola Panamericana. 11 p.

Freire Coba, R.P. 2011. Tratamiento profiláctico con Ozono y Lidocaína al $0.5 \%$ intrauterino en las primeras 12 horas posparto sobre las infecciones uterinas en ganado lechero en Zamorano. Proyecto Especial del Programa de Ing. Agr., Zamorano, Honduras, Escuela Agrícola Panamericana. $31 \mathrm{p}$. 
Gallozzi Cálix, R. 1998. Evaluación de un plan de capacitación sobre sanidad animal dirigido a productores pecuarios de cuatro comunidades de la región del Yeguare. Proyecto Especial del Programa de Ing. Agr., Zamorano, Honduras, Escuela Agrícola Panamericana. $30 \mathrm{p}$.

García Guerrero, B.A. 2005. Efecto de la PG 600 en la inducción del estro y la prolificidad en cerdas posdestete. Proyecto Especial del Programa de Ing. Agr., Zamorano, Honduras, Escuela Agrícola Panamericana. $14 \mathrm{p}$.

Gonzáles Monroy, M. 1994. Dinámica poblacional y parasitismo de la mosca doméstica, Musca domestica, y la mosca del establo, Stomoxys calcitrans. Proyecto Especial del Programa de Ing. Agr., Zamorano, Honduras, Escuela Agrícola Panamericana. s.p.

Guerrero Soria, S.C. 2006. Caracterización de los cinco principales parásitos gastrointestinales y el efecto de la aplicación de ivermectina + praziquantel en equinos en la región de la Sierra Central, Ecuador. Proyecto Especial del Programa de Ing. Agr., Zamorano, Honduras, Escuela Agrícola Panamericana. 23 p.

Guevara Florentino, F. 2008. Evaluación de un programa de sincronización y resincronización de celos en vacas lecheras con anestro posparto. Proyecto Especial del Programa de Ing. Agr., Zamorano, Honduras, Escuela Agrícola Panamericana. 9 p.

Guevara López, L. 2007. Efecto de la ozonoterapia sobre los parámetros hematológicos y constantes fisiológicas en equinos. Proyecto Especial del Programa de Ing. Agr., Zamorano, Honduras, Escuela Agrícola Panamericana. $29 \mathrm{p}$.

Gutiérrez Luna, A.J. y M.S. Borjas Carvajal. 2010. Evaluación de las Ivermectinas al 4\% LP (Liberación Programada), 3.15\% (Tixotrópica) y la Doramectina al $1 \%$ sobre el control de endo y ectoparásitos y en la ganancia de peso en vaquillas de levante. Proyecto Especial del Programa de Ing. Agr., Zamorano, Honduras, Escuela Agrícola Panamericana. 15 p.

Guzmán Calderón, J. 2002. Caracterización de la flora bacteriana presente en las heces de los lechones de la sección de maternidad de Zamorano, Honduras. Proyecto Especial del Programa de Ing. Agr., Zamorano, Honduras, Escuela Agrícola Panamericana. $39 \mathrm{p}$.

Herrarte Abascal, M.E. 2005. Detección temprana de preñez con ultrasonido de tiempo real en bovinos. Proyecto Especial del Programa de Ing. Agr., Zamorano, Honduras, Escuela Agrícola Panamericana. 21 p.
López Rodríguez, A. 2000. Perfil metabólico del hato lechero de Zamorano. Proyecto Especial del Programa de Ing. Agr., Zamorano, Honduras, Escuela Agrícola Panamericana. $30 \mathrm{p}$.

Martínez Pittí, C.B. y I.F. Sierra Sagastume. 2010. Efecto de la aplicación de eCG al momento del retiro del implante intravaginal DIV-B ${ }^{\circledR}$ sobre los porcentajes de inducción de celo y preñez en vacas lecheras con anestro pos parto. Proyecto Especial del Programa de Ing. Agr., Zamorano, Honduras, Escuela Agrícola Panamericana. $17 \mathrm{p}$.

Matamoros Hernández, J.E. y J.G. Moreno Rajo. 2009. Efecto de la aplicación de Calfosvit $\mathrm{Se}^{\circledR}$ sobre el comportamiento reproductivo de vacas lecheras. Proyecto Especial del Programa de Ing. Agr., Zamorano, Honduras, Escuela Agrícola Panamericana. $17 \mathrm{p}$.

Medina Ortega, M. 1999. Evaluación de los niveles séricos de PGFM durante el período de puerperio temprano y su relación con los parámetros reproductivos en hembras bovinas. Proyecto Especial del Programa de Ing. Agr., Zamorano, Honduras, Escuela Agrícola Panamericana. $18 \mathrm{p}$.

Mendoza García, J. 2001. Caracterización de los sistemas de producción porcina e incidencia de parásitos gastrointestinales en seis granjas del Valle Yeguare, Honduras. Proyecto Especial del Programa de Ing. Agr., Zamorano, Honduras, Escuela Agrícola Panamericana. $15 \mathrm{p}$.

Menjívar Polanco, R.J. y E. Barahona Rosales. 2009. Efecto de los implantes intravaginales nuevos o usados y de dos tiempos de retiro sobre el porcentaje de preñez en vacas de carne. Proyecto Especial del Programa de Ing. Agr., Zamorano, Honduras, Escuela Agrícola Panamericana. $14 \mathrm{p}$.

Mercadal Zapata, J. 1994. Diagnóstico subclínico de metritis utilizando un metabolito de prostaglandina. Proyecto Especial del Programa de Ing. Agr., Zamorano, Honduras, Escuela Agrícola Panamericana. 56 p.

Montalván Ruíz, J. 2004. Efecto la suplementación con grasa protegida en la dieta sobre el desempeño reproductivo en ganado lechero. Proyecto Especial del Programa de Ing. Agr., Zamorano, Honduras, Escuela Agrícola Panamericana. $14 \mathrm{p}$.

Morales del Cid, I. 2004. Efecto de la Lidocaína 1\% en el tratamiento profiláctico de la metritis puerperal séptica y la retención placentaria durante el periodo del puerperio en ganado lechero. Proyecto Especial del Programa de Ing. Agr., Zamorano, Honduras, Escuela Agrícola Panamericana. 19 p. 
Morán Berlioz, A. 1998. Efecto de las micotoxinas sobre la producción y reproducción en el hato lechero del Zamorano. Proyecto Especial del Programa de Ing. Agr., Zamorano, Honduras, Escuela Agrícola Panamericana. $20 \mathrm{p}$.

Morán Ramírez, K. y M. Díaz Lezama. 2006. Aplicación de la Prueba de Resistencia Hiperosmótica (PRH) para predecir la congelabilidad del semen bovino. Proyecto Especial del Programa de Ing. Agr., Zamorano, Honduras, Escuela Agrícola Panamericana. 13 p.

Orellana Banegas, J. y E. Peralta Peralta. 2007. Manual de procedimientos para el laboratorios de transferencia de embriones en bovinos de la empresa Genetic Resources International (GRI) and Sexing Technologies. Proyecto Especial del Programa de Ing. Agr., Zamorano, Honduras, Escuela Agrícola Panamericana. 42 p.

Ortega Obando, M.S. 2003. Efecto de la lidocaína en el tratamiento de la retención placentaria y metritis puerperal en ganado lechero. Proyecto Especial del Programa de Ing. Agr., Zamorano, Honduras, Escuela Agrícola Panamericana. 33 p.

Osorio Estévez, J. 2004. Evaluación de extracto acuoso Rhizophora mangle y Lidocaína al $1 \%$ en el tratamiento de la metritis puerperal y el reinicio de la actividad ovárica posparto en ganado lechero. Proyecto Especial del Programa de Ing. Agr., Zamorano, Honduras, Escuela Agrícola Panamericana. 21 p.

Pazmiño Haro, M. 2005. Efecto del amamantamiento restringido y manipulación uterina sobre el reinicio de la actividad ovárica posparto en ganado de carne. Proyecto Especial del Programa de Ing. Agr., Zamorano, Honduras, Escuela Agrícola Panamericana. $18 \mathrm{p}$.

Pérez de La Ossa, J. 2007. Tasa de preñez en vacas con dispositivos intravaginales CIDR nuevo y usados dos o tres veces por siete días, en la Hacienda Santa Elisa, El Paraíso, Honduras. Proyecto Especial del Programa de Ing. Agr., Zamorano, Honduras, Escuela Agrícola Panamericana. 11 p.

Perla Barrera, O. 2005. Efecto profiláctico del ozono aplicado vía intrauterina en la etapa posparto comparado con la Lidocaína y PGF2 $\alpha$ sobre el comportamiento reproductivo del ganado lechero. Proyecto Especial del Programa de Ing. Agr., Zamorano, Honduras, Escuela Agrícola Panamericana. $20 \mathrm{p}$.
Pinto Portillo, M.T. y M.R. Chacón García. 2009. Comparación de las concentraciones plasmáticas de progesterona en vacas implantadas con dispositivos intravaginales y vacas gestantes. Proyecto Especial del Programa de Ing. Agr., Zamorano, Honduras, Escuela Agrícola Panamericana. 21 p.

Ponce Velásquez, E.A. y J.J. Marín Elías. 2002. Detección de anticuerpos contra Leucosis Bovina Enzoótica, Rinotraqueitis Infecciosa Bovina, Brucelosis Bovina y efecto de estas enfermedades en la producción del hato lechero de Zamorano, Honduras. Proyecto Especial del Programa de Ing. Agr., Zamorano, Honduras, Escuela Agrícola Panamericana. $47 \mathrm{p}$.

Portillo Sandoval, I. y J. Villacís Carrera. 2005. Efecto de la profilaxis posparto con Lidocacína al $1 \%$ y $\mathrm{PGF}_{2} \alpha$ sobre el comportamiento reproductivo del ganado lechero en Zamorano. Proyecto Especial del Programa de Ing. Agr., Zamorano, Honduras, Escuela Agrícola Panamericana. $16 \mathrm{p}$.

Posas Flores, A. 2002. Determinación del nivel de anticuerpos contra parvovirus y factores de riesgo en seis granjas porcinas de Honduras. Proyecto Especial del Programa de Ing. Agr., Zamorano, Honduras, Escuela Agrícola Panamericana. 34 p.

Raudales Zúñiga, J. y H. Cerrato Zelaya. 2006. Evaluación de dos diluyentes para la criopreservación de semen bovino. Proyecto Especial del Programa de Ing. Agr., Zamorano, Honduras, Escuela Agrícola Panamericana. $20 \mathrm{p}$.

Revelo Saltos, X. 2005. Efecto de la aplicación de hierro dextrán a terneros Holstein, Jersey y sus cruces, recién nacidos, sobre su crecimiento en las primeras siete semanas de vida. Proyecto Especial del Programa de Ing. Agr., Zamorano, Honduras, Escuela Agrícola Panamericana. $16 \mathrm{p}$.

Rodas Lagos, R. 1997. Evaluación del Apazote (Chenopodium ambrosioides) en el control de endoparásitos en ovejas. Proyecto Especial del Programa de Ing. Agr., Zamorano, Honduras, Escuela Agrícola Panamericana. 26 p.

Rodríguez Calvo, M.A. y M.R. Lobo Rosa. 2011. Porcentaje de preñez en vacas lecheras tratadas con implantes intravaginales y dos fuentes comerciales de eCG. Proyecto Especial del Programa de Ing. Agr., Zamorano, Honduras, Escuela Agrícola Panamericana. $21 \mathrm{p}$. 
Rodríguez, L. 2000. Efectividad de la vacuna recombinante Bm86 en el control de la garrapata Boophilus microplus. Proyecto Especial del Programa de Ing. Agr., Zamorano, Honduras, Escuela Agrícola Panamericana.

Ruíz Faz, D.A. y V.H. Ibarra Ruíz. 2005. Efecto del óxido de calcio $(\mathrm{CaO})$ sobre el contenido químico y microbiológico de la cama y su efecto en la producción de pollos de engorde. Proyecto Especial del Programa de Ing. Agr., Zamorano, Honduras, Escuela Agrícola Panamericana. $19 \mathrm{p}$.

Sierra Méndez, J. 2005. El pH urinario, el pH en el estiércol y la temperatura rectal como indicadores de hipocalcemia, acidosis ruminal o infecciones uterinas en vacas en transición. Proyecto Especial del Programa de Ing. Agr., Zamorano, Honduras, Escuela Agrícola Panamericana. $14 \mathrm{p}$.

Sobalvarro Acosta, M. 2003. Estudio epidemiológico de Rinotraqueitis Infecciosa Bovina y Diarrea Viral Bovina en 10 explotaciones ganaderas de Honduras. Proyecto Especial del Programa de Ing. Agr., Zamorano, Honduras, Escuela Agrícola Panamericana. $55 \mathrm{p}$.

Sosa Cuevas, J.A. 2009. Prevalencia de la leptospirosis bovina en 23 explotaciones ganaderas en Honduras. Proyecto Especial del Programa de Ing. Agr., Zamorano, Honduras, Escuela Agrícola Panamericana. $24 \mathrm{p}$.

Vázcones Miño, X. y J.A. Ortega González. 2009. Efecto de dos dosis de eCG al momento de retirar los implantes intravaginales DIV-B ${ }^{\circledR}$ sobre el porcentaje de preñez en ganado bovino. Proyecto Especial del Programa de Ing. Agr., Zamorano, Honduras, Escuela Agrícola Panamericana. $14 \mathrm{p}$.
Velázquez Mejía, D y G.J. Vélez Bravo. 2011. Porcentaje de preñez en vacas con baja condición corporal tratadas con dos dosis de eCG en el día ocho del tratamiento con dispositivos intravaginales DIV-B ${ }^{\circledR}$. Proyecto Especial del Programa de Ing. Agr., Zamorano, Honduras, Escuela Agrícola Panamericana. $16 \mathrm{p}$.

Vélez Pérez, S. 2005. Sincronización de celos e inseminación artificial a tiempo fijo en ganado de carne en la Hacienda Cuba, Montelíbano, Colombia. Proyecto Especial del Programa de Ing. Agr., Zamorano, Honduras, Escuela Agrícola Panamericana. $28 \mathrm{p}$.

Vergara Icaza, Y.L. 2005. Relación entre el diámetro del folículo pre-ovulatorio y el porcentaje de preñez en ganado lechero. Proyecto Especial del Programa de Ing. Agr., Zamorano, Honduras, Escuela Agrícola Panamericana. $23 \mathrm{p}$.

Villavicencio Celi, P.A. 2007. Respuesta de dos razas cebuínas y un cruce comercial a la inducción y sincronización del celo utilizando el dispositivo intravaginal de liberación programada $\mathrm{CIDR}^{\circledR}$. Proyecto Especial del Programa de Ing. Agr., Zamorano, Honduras, Escuela Agrícola Panamericana. $13 \mathrm{p}$.

Recibido para publicación el 16 de abril de 2012.

Aceptado para publicación el 21 de septiembre de 2012. 